\title{
Study of Resistive Superconducting Transition of Thallium Based Superconductors
}

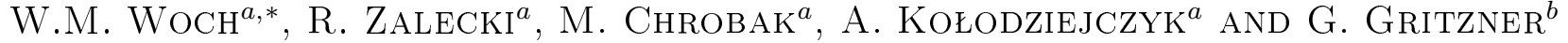 \\ ${ }^{a}$ Solid State Physics Dept., AGH University of Science and Technology \\ al. Mickiewicza 30, PL 30-059, Kraków, Poland \\ ${ }^{b}$ Institute for Chemical Technology of Inorganic Materials, Johannes Kepler University, A 4040 Linz, Austria
}

\begin{abstract}
A width and shape of resistive transition as well as thermal fluctuations of the thallium based superconductors were analyzed theoretically. The applied magnetic field widens the resistive transition according to the following formula: $\Delta T=C H^{m}+\Delta T_{0}$. The exponent $m$ fluctuates around $2 / 3$ and depends on vortex structure and strength of the pinning force. The shape of the resistive transition was fitted by two models: the first one based on Ambegaokar-Halperin theory and the other one based on Anderson-Kim theory expressed by the exponential formula. Critical fluctuations were analyzed on the basis of magnetoresistance measurements results. The critical exponents have been calculated above the critical temperature $T_{\mathrm{c}}$ as well as for temperatures close to the zero resistance critical temperature.
\end{abstract}

PACS: 74.72-h, 74.40n, 74.20.De, 74.25.Ha

\section{Introduction}

The resistive transition from the normal to the superconducting state of high temperature superconductors hides many interesting physical phenomena. In classical - low temperature - superconductors the resistive transition is generally sharp and a significant widening of the transition is usually hardly observable. This is because in the low temperature superconductors pinning is reasonably effective while the effects of fluctuations are relatively unimportant, so there is rather a well defined sharp resistive transition in which the resistance drops to zero in a narrow temperature range [1]. However, an applied magnetic field can broaden the resistive transition and can shift the resistive transition to lower temperatures. A quite different behavior can be observed in high temperature superconductors (HTS) in which the width of the resistive transition can be easily observed, and it is one of the important parameters characterizing the superconducting state. The behavior of resistance in applied magnetic fields shows an anomalous broadening, in particular when the magnetic field is applied along the $c$-axis (see Fig. 1). This phenomenon has been interpreted in the frame of the vortex structure and flux motion mechanism [2-5]. There are two models of the broadening effect: the first one based on Ambegaokar-Halperin theory that describes the resistive transitions by the modified Bessel function [6, 7] and the other one based on Anderson-Kim theory expressed by the exponential formula of the field dependence of the resistance $[8,9]$.

In this paper we study the width and the shape of the resistive transitions as well as the thermal fluctuation of the thallium based superconductors. Thal-

* corresponding author; e-mail: wmwoch@agh.edu.pl

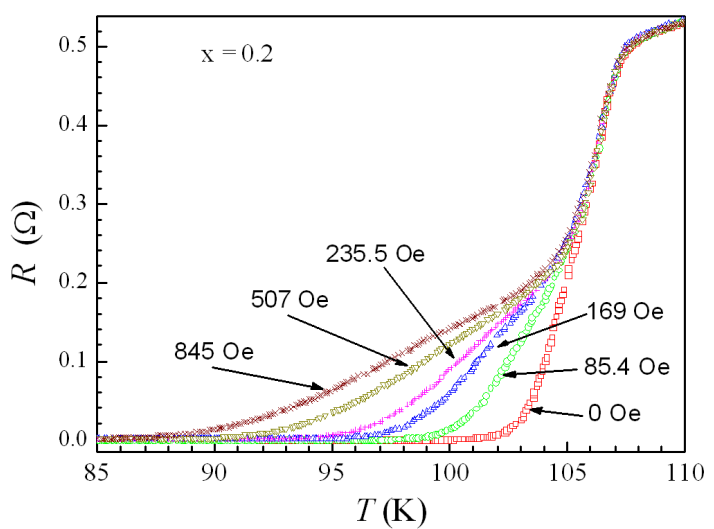

Fig. 1. Magnetoresistance as a function of temperature of $\left(\mathrm{Tl}_{0.5} \mathrm{~Pb}_{0.5}\right) \mathrm{Sr}_{2}\left(\mathrm{Ca}_{1-x} \mathrm{Gd}_{x}\right) \mathrm{Cu}_{2} \mathrm{O}_{z}$ for $x=0.2$.

lium based superconductors are still very interesting not only for the transport of electric energy but also for applications in the field of superconductor digital electronics such as switches, magnetic shields, resonators for microwave filters, microwave guides, antennas and other devices. A research on superconductors for the power industry is mainly focusing on superconducting tapes which are expected to replace bismuth based powder in tube tapes and coated conductors - the second generation of high temperature superconducting tapes. For this study we have chosen following thallium based superconductors. The first one is a $c$-axis oriented and $a-b$ aligned superconducting film $\left(\mathrm{Tl}_{0.6} \mathrm{~Pb}_{0.24} \mathrm{Bi}_{0.16}\right)\left(\mathrm{Sr}_{0.9} \mathrm{Ba}_{0.1}\right)_{2} \mathrm{Ca}_{2} \mathrm{Cu}_{3} \mathrm{O}_{y}$ obtained by the screen printing method on $\mathrm{LaAlO}_{3}$ substrate [10]. The critical temperature of this sample is $T_{\mathrm{c}}=112 \mathrm{~K}$, the critical current is $J_{c} \approx 10^{6} \mathrm{~A} / \mathrm{cm}^{2}$ at $77 \mathrm{~K}$ in the self field and the irreversibility fields are 


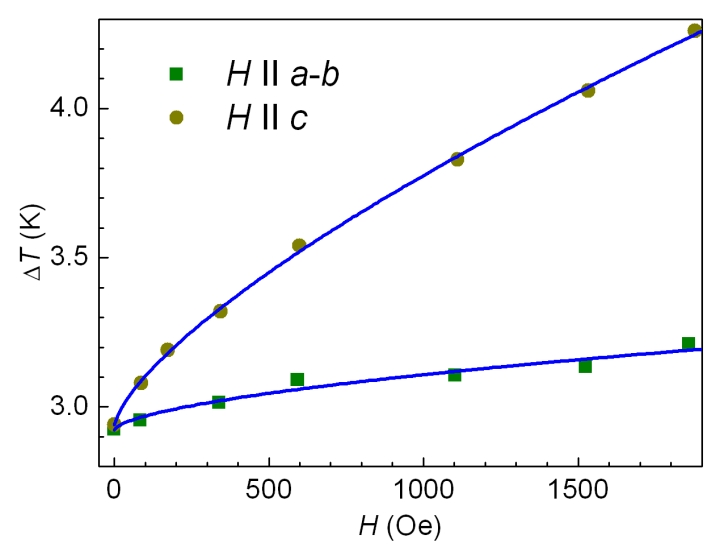

Fig. 2. Width of the resistive transition of a $\left(\mathrm{Tl}_{0.6} \mathrm{~Pb}_{0.24} \mathrm{Bi}_{0.16}\right)\left(\mathrm{Ba}_{0.1} \mathrm{Sr}_{0.9}\right)_{2} \mathrm{Ca}_{2} \mathrm{Cu}_{3} \mathrm{O}_{y}$ film on single-crystalline (100) lanthanum aluminate as a function of the applied magnetic field for two magnetic field orientations: $H \| a-b$ plane (squares) and $H \| c$-axis (circles). Solid lines are the fit to Eq. (8).

$H_{\text {irr }}=98 \mathrm{~T}$ for $H \| a-b$ and $H_{\text {irr }}=4.1 \mathrm{~T}$ for $H \| c$ at $T=77 \mathrm{~K}[11,12]$. The second one is superconducting $\left(\mathrm{Tl}_{0.5} \mathrm{~Pb}_{0.5}\right)\left(\mathrm{Sr}_{0.85} \mathrm{Ba}_{0.15}\right)_{2} \mathrm{Ca}_{2} \mathrm{Cu}_{3} \mathrm{O}_{z}$ film prepared on highly polished, untextured silver substrate [13]. A superconducting transition temperature of this specimen is $T_{\mathrm{c}, 50 \%}=114.7 \mathrm{~K}$ and the critical current is $J_{\mathrm{c}}=11.4 \times$ $10^{3} \mathrm{~A} / \mathrm{cm}^{2}$ at $77 \mathrm{~K}$ in the self field. The irreversibility fields of this film are very small and they are as follows: $H_{\text {irr }}=80$ Oe for $H \| a-b$ and $H_{\text {irr }}=20$ Oe for $H \| c$ at $T=$ $77 \mathrm{~K}$ [14-16]. The last class of superconductors studied in this paper are bulk $\left(\mathrm{Tl}_{0.5} \mathrm{~Pb}_{0.5}\right) \mathrm{Sr}_{2}\left(\mathrm{Ca}_{1-x} \mathrm{Gd}_{x}\right) \mathrm{Cu}_{2} \mathrm{O}_{z}$ with $x=0.1,0.2$ and $0.3[17]$. These superconductors have been studied in several papers [18-20].

\section{Width of the resistive transition}

The temperature width of the resistive transitions of HTS was introduced by M. Tinkham [7] who extended the model of giant flux creep proposed by Yeshurun and Malozemoff [21]. According to Ambegaokar and Helperin in the limit of the small currents the resistance of the superconducting Josephson weak links can be described as follows [6]:

$$
\frac{R}{R_{\mathrm{n}}}=\left(P_{0}\left(\frac{\gamma}{2}\right)\right)^{-2},
$$

where $P_{0}$ is the modified Bessel function (see Eq. (9)), $R$ is the resistance of the weak links, $R_{\mathrm{n}}$ is the resistance in the normal state at a given temperature and $\gamma$ is the parameter related to the activation energy $U_{0}$ which must be overcome to allow flux motion and hence resistance. Using heuristic scaling argument Yeshurun and Malozemoff have proposed that the activation energy $U_{0}$ should have a form:

$$
U_{0}=\frac{\beta H_{c}^{2} \xi \varphi_{0}}{B}
$$

where $H_{\mathrm{c}}$ is the thermodynamic critical field, $\xi$ is the co- herence length, $\varphi_{0}$ is the flux quantum $h c / 2 e, B$ is the flux density in the sample, and the parameter $\beta \approx 1$ is introduced to absorb all numerical factors. Using the substitution of Ginzburg-Landau relations: $\varphi_{0}=$ $2 \sqrt{2} \pi H_{c} \xi \lambda$ and $J_{c 0}=c H_{c} / 3 \sqrt{6} \pi \lambda$ one can obtain:

$$
U_{0}=\left(\frac{3 \sqrt{3} \varphi_{0}^{2} \beta}{2 c}\right)\left(\frac{J_{c 0}}{B}\right) .
$$

This formula involves only one materials parameter $J_{\mathrm{c} 0}$ instead of two $\left(H_{\mathrm{c}}\right.$ and $\left.\xi\right)$ in formula (2).

Since the thermally activated process depends on $U_{0} / k_{\mathrm{B}} T$, one can define the normalized height barrier as follows [7]:

$$
\gamma=\frac{U}{k_{\mathrm{B}} T}=\left(\frac{C J_{c 0}(0)}{T_{c} B}\right) g(t),
$$

where the function $g(t)$ is the parameter of GinzburgLandau free energy expansion, $t=T / T_{\mathrm{c}}$. Thus, Eq. (4) can be described in the form:

$$
\gamma=\frac{U}{k_{\mathrm{B}} T}=\frac{A(1-t)^{3 / 2}}{H},
$$

where the coefficient $A$ depends on the critical current at zero magnetic field and $H$ is the applied magnetic field. Putting Eq. (5) into Eq. (1) and taking only two first terms of the modified Bessel series, the following equation for the magnetic field $H \neq 0$ may be derived:

$$
\begin{aligned}
\frac{R}{R_{\mathrm{n}}} & =\left(P_{0}\left(\frac{A(1-t)^{3 / 2}}{2 H}\right)\right)^{-2} \\
& =\left(1-\left(\frac{A(1-t)^{3 / 2}}{4 H}\right)^{2}\right)^{-2} .
\end{aligned}
$$

Equation (6) is valid for each value of $R / R_{\mathrm{n}}$. If we consider $R / R_{n}=0$, then a simple calculation yields the following result for the width of resistive transition:

$$
\Delta T=16 T_{\mathrm{C}}\left(\frac{1}{A}\right)^{2 / 3} H^{2 / 3}=C H^{2 / 3} .
$$

For HTS the resistive transition is relatively wide even for $H=0$, so $\Delta T(H=0) \neq 0$. Therefore, Eq. (7) should be rewritten as [11, 12]:

$$
\Delta T=C H^{m}+\Delta T_{0},
$$

where $m=2 / 3=1 / n$ and $\Delta T_{0}$ is the width of the resistive transition at the zero applied magnetic field. The exponent $n=3 / 2$ comes from the irreversibility fields of the HTS [22] originally introduced by Müller, Takashige and Bednorz [23]. This exponent could fluctuate around this value as a function of the vortex structure and the strength of the pinning force. Equation (8) has only two unknown parameters: $C$ and $m$. The width of the resistive transition at the zero applied magnetic field $\Delta T_{0}$ is usually taken from experiment.

The magnetic field dependencies of the width of the resistive transition of thallium films show large anisotropy (see for instance Fig. 2). One can notice significant differences between the width of the resistive transitions for $H \| a-b$ and for $H \| c$. The fit procedure gave the 
exponents which are $m=0.59 \pm 0.02$ for $H \| a-b$ and $m=0.71 \pm 0.02$ for $H \| c$. The values of exponent $m$ less than $2 / 3$ indicates that a strong pinning and a vortex glass structure exists (the applied field is parallel to the $a-b$ plane). On the other hand, higher than $2 / 3$ values of $m$ suggest the pinning force is weak and the vortex structure is like in HTS. These results are consistent with the temperature dependence of the critical currents and of the irreversibility fields $[15,16]$.

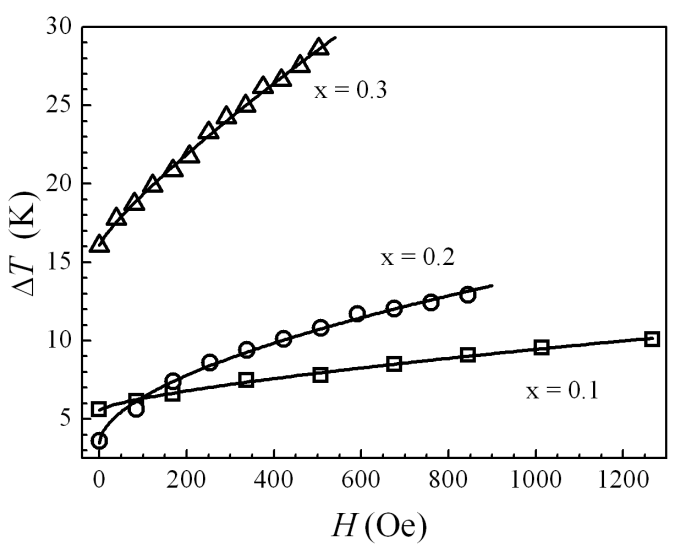

Fig. 3. Width of the resistive transition of $\left(\mathrm{Tl}_{0.5} \mathrm{~Pb}_{0.5}\right) \mathrm{Sr}_{2}\left(\mathrm{Ca}_{1-x} \mathrm{Gd}_{x}\right) \mathrm{Cu}_{2} \mathrm{O}_{z} \quad$ superconductors with $x=0.1$ (open squares) $x=0.2$ (open circles) and $x=0.3$ (open triangles). Solid lines are the fit to Eq. (8).

The interesting features of the broadening of the resistive transition may be observed in the $\left(\mathrm{Tl}_{0.5} \mathrm{~Pb}_{0.5}\right) \mathrm{Sr}_{2}\left(\mathrm{Ca}_{1-x} \mathrm{Gd}_{x}\right) \mathrm{Cu}_{2} \mathrm{O}_{z}$ superconductors (see Fig. 3). The sample with $x=0.2$ features the best superconducting parameters like critical temperature, critical current and irreversibility field. Nevertheless a magnetic broadening of the resistive transition is smallest for the sample with $x=0.1$. One can observe the crossover of the dependencies $\Delta T(H)$ for $x=0.1$ and $x=0.2$ that can be explained by the higher magnetic moment of gadolinium in the external magnetic field of the sample with $x=0.2$. The fitted exponents show that only $\left(\mathrm{Tl}_{0.5} \mathrm{~Pb}_{0.5}\right) \mathrm{Sr}_{2}\left(\mathrm{Ca}_{1.8} \mathrm{Gd}_{0.2}\right) \mathrm{Cu}_{2} \mathrm{O}_{z}$ sample exhibits strong pinning and glassy vortex state with $n=0.56$. Also the highest critical current is observed in this sample. The fits as well as the experimentally obtained parameters of $\left(\mathrm{Tl}_{0.5} \mathrm{~Pb}_{0.5}\right) \mathrm{Sr}_{2}\left(\mathrm{Ca}_{1-x} \mathrm{Gd}_{x}\right) \mathrm{Cu}_{2} \mathrm{O}_{z}$ superconductors are summarized in the Table.

\section{Transition shape}

The shape of the temperature and the field dependent resistive transition was analyzed using the two models: first, based on the Ambegaokar-Halperin theory $(\mathrm{AH})[6,7]$ and the second, based on the AndersonKim theory $(\mathrm{AK})[8,9]$. The results were obtained for $\left(\mathrm{Tl}_{0.6} \mathrm{~Pb}_{0.24} \mathrm{Bi}_{0.16}\right)\left(\mathrm{Ba}_{0.1} \mathrm{Sr}_{0.9}\right)_{2} \mathrm{Ca}_{2} \mathrm{Cu}_{3} \mathrm{O}_{y}$ film on singlecrystalline lanthanum aluminate substrate.

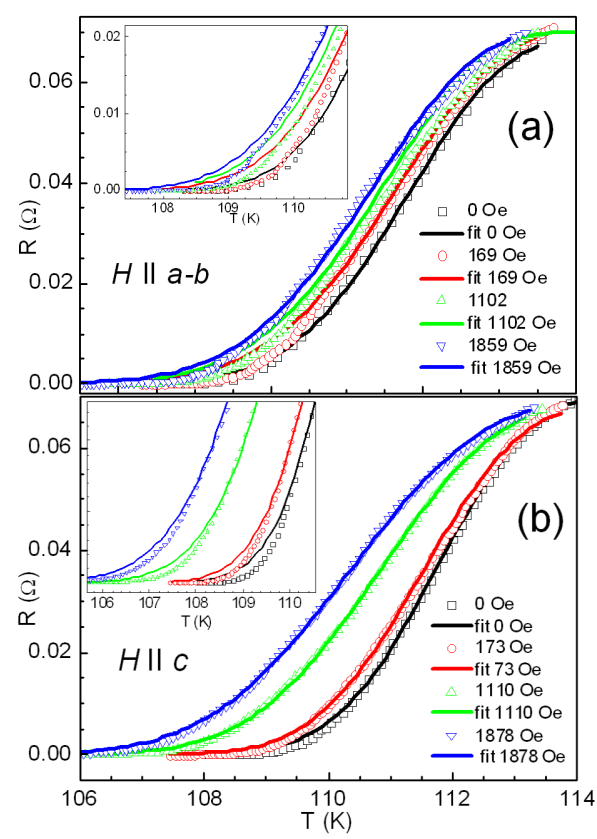

Fig. 4. Resistance vs. temperature of a $\left(\mathrm{Tl}_{0.6} \mathrm{~Pb}_{0.24} \mathrm{Bi}_{0.16}\right)\left(\mathrm{Ba}_{0.1} \mathrm{Sr}_{0.9}\right)_{2} \mathrm{Ca}_{2} \mathrm{Cu}_{3} \mathrm{O}_{y}$ film for (a) magnetic fields $H \| a-b$ and (b) magnetic field $H \| c$ [2]. Solid lines are the fitting of Eq. (13) (AK model). Insets shows $R(T)$ close to $T_{\mathrm{c} 0}$ in enlarged scale.

TABLE

Superconducting transition temperatures $T_{\mathrm{c} 50 \%}$ and the transition width $\Delta T_{90 \%-10 \%}$ of $\left(\mathrm{Tl}_{0.5} \mathrm{~Pb}_{0.5}\right) \mathrm{Sr}_{2}\left(\mathrm{Ca}_{1-x} \mathrm{Gd}_{x}\right) \mathrm{Cu}_{2} \mathrm{O}_{z}$ samples obtained from resistance measurements. The fit parameters $\Delta T_{0}$, $n$ and $C$ are obtained using Eq. (8).

\begin{tabular}{c|c|c|c|c|c}
\hline \hline$x$ & $T_{\mathrm{c} 50 \%}$ & $\Delta T_{90 \%-10 \%}$ & $\Delta T_{0}$ & $m$ & $C$ \\
\cline { 2 - 4 } & \multicolumn{3}{|c|}{$[\mathrm{K}]$} & & \\
\hline 0.1 & 101.1 & 5.6 & $5.58 \pm 0.13$ & $0.72 \pm 0.05$ & $0.027 \pm 0.011$ \\
\hline 0.2 & 105.3 & 3.6 & $3.5 \pm 0.3$ & $0.56 \pm 0.03$ & $0.22 \pm 0.05$ \\
\hline 0.3 & 98.4 & 16.3 & $16.08 \pm 0.18$ & $0.84 \pm 0.03$ & $0.069 \pm 0.014$
\end{tabular}

According to the first model, in the limit of the small currents the resistance of each weak link treated as a Josephson junction is reduced by the ratio expressed by the Eq. (1), where $P_{0}$ is the modified Bessel function given by:

$$
P_{0}(x)=\sum_{s=0}^{\infty} \frac{1}{(s !)^{2}}\left(\frac{x}{2}\right)^{2 s},
$$

and parameter $\gamma$ is determined by Eq. (5). This means that the activation energy depends only on the temperature $T$ and the applied magnetic field $H$.

Finally the full Eq. (6) was used to fit the shape of the resistive transition in $\mathrm{AH}$ model. This equation has only two parameters: $A$ and $T_{\mathrm{c}}$. The onset resistance $R_{\mathrm{n}}$ may be taken from experiment.

According to Anderson and Kim theory [8, 9] the resistance due to the flux motion in the mixed state of an epitaxial superconducting film can be well described by 
thermal activation formula:

$$
R=R_{0} \exp \left(-U / k_{B} T\right) .
$$

The activation energy $U$ has a functional dependence on temperature $T$, magnetic field $H$ and transport current $I$ :

$$
U=A^{*} H^{-\beta} \ln \left(I / I_{0}\right) g^{*}(T),
$$

where $A^{*}$ is a constant depending weakly on applied magnetic field, $\beta$ is a dimensionless constant close to $1, I_{0}$ is the threshold current. $g^{*}(T)$ is a temperature function consisting of the temperature dependences of the critical field and the penetration depth given by [7]:

$$
g^{*}(t)=\left(1-t^{2}\right)\left(1-t^{4}\right)^{1 / 2} \text {. }
$$

Combining Eqs. (10), (11) and (12) one can recast the dissipation due to thermally activated flux creep with field, current, and temperature as follows:

$$
R=R_{0}\left(\frac{I}{I_{0}}\right)^{\frac{-A^{*} H^{-\beta}\left(1-t^{2}\right)\left(1-t^{4}\right)^{p}}{k^{T}}},
$$

where we have introduced the more general parameter $p$ instead of $1 / 2$.

For initial fitting some starting values of the parameters should be estimated using special conditions of the experimental setup. Then only two parameters $A^{*}$ and $T_{\mathrm{c}}$ can be varied in order to obtain the best fit [24]. The shape of temperature and field dependent resistive transition was interpreted in terms of the Eqs. (6) and (13). The fits using Eq. (13) describe the measurements of the resistance slightly better than Eq. (6) and are shown in Fig. 4. The reason is that the AK theory includes the current dependence of activation energy. The calculated critical temperatures for both models are very close to the onset temperature $T_{\mathrm{c}}^{\mathrm{onset}}=115.2 \mathrm{~K}$ of this superconductor and can be treated as a freezing temperature $T_{\mathrm{g}}$ of the vortex-glass state. The surprising fact is that the freezing temperature does not depend on the applied magnetic field within the measured range. The fitting procedures show that the parameters $A$ and $A^{*}$ strongly depend on the applied magnetic field.

\section{Thermal fluctuations}

A very short coherence length as well as the large anisotropy of HTS affect the thermal fluctuations around the superconducting transition. Within the critical region the competition between the critical fluctuations at lower temperatures and the gaussian (stochastic) fluctuations at higher temperatures the critical temperatures exists and depend on both the applied magnetic field and pressure [25-27]. The gaussian fluctuations can give the information on the effective dimensionality of superconductivity in HTS materials. Because of a huge anisotropy the superconductors usually show the three-dimensional (3D) to the two-dimensional (2D) transition in the critical region for polycrystalline, single crystal as well as for thin film samples [24, 28-30]. To analyze the thermal fluctuation of the thallium based superconductors the formula for a field dependent conductivity within the transition region was used [31]:

$$
\Delta \sigma=K \varepsilon^{-\lambda}
$$

where $\varepsilon=\left(T-T_{c}\right) / T_{c}, \lambda$ is the critical exponent and $K$ is a constant. The temperature dependence of the excess conductivity is defined within the Ginzburg-Landau mean field approximation [32]:

$$
\Delta \sigma(T)=\frac{1}{R(T)}-\frac{1}{R_{R}(T)},
$$

where $R(T)$ is the measured resistance and $R_{R}(T)$ is the resistance obtained by the linear extrapolation of resistance data from around $170 \mathrm{~K}$, the highest measured temperature, down to temperatures below $T_{\mathrm{c}}$ [33].

To get $\Delta \sigma$ we have determined the $R_{\mathrm{R}}$ for temperatures near $T_{\mathrm{c}}$ by linear extrapolation from higher to lower temperatures of $R_{\mathrm{R}}(T)$ as follows:

$$
R_{R}(T)=R_{0}+\left(\frac{\mathrm{d} R}{\mathrm{~d} T}\right) T
$$

where $R_{0}$ and $\mathrm{d} R / \mathrm{d} T$ are constants. The linearity of $R_{\mathrm{R}}(T)$ is observed in all the measurements above $130 \mathrm{~K}$.

In the critical region, where the critical fluctuations are observed the full dynamical scaling theory [34] predicts that the excess conductivity (Eq. (15)) diverges at the critical temperature with the critical exponent given by the relation:

$$
\lambda=\nu(2+z-d+\eta),
$$

where $\nu$ is the critical exponent for the coherence length, $z$ is the dynamical exponent, $d$ is the dimensionality of fluctuation spectrum and $\eta$ is exponent for the order parameter of the correlation function. The thermodynamic properties of superconductors in the critical region are the same as a for 3D-XY model. Then one should substitute $\nu=2 / 3, z \cong 3 / 2$ and $\eta \cong 0$.

In the regimes away from the critical temperature the critical exponents are dominated by Gaussian fluctuations. The mean field Ginzburg-Landau theory predicts that $\nu=1 / 3, z=2$ and $\eta=0$. Then as shown by Aslamasov and Larkin [31] the gaussian fluctuations can be described by:

$$
\lambda=2-\frac{d}{2},
$$

where $d$ is the dimensionality of fluctuation spectrum.

To determine the critical exponents we plotted the temperature dependence of the following expression:

$$
-\left(\frac{\mathrm{d}}{\mathrm{d} T} \ln \left(\frac{1}{R}-\frac{1}{R_{R}}\right)\right)^{-1}=\frac{1}{\lambda}\left(T-T_{c}\right) .
$$

The critical exponent is given by reversal slope of the curve within a linear regions of the temperature.

The results of Eq. (19) for $\left(\mathrm{Tl}_{0.6} \mathrm{~Pb}_{0.24} \mathrm{Bi}_{0.16}\right)\left(\mathrm{Ba}_{0.1} \mathrm{Sr}_{0.9}\right)_{2} \mathrm{Ca}_{2} \mathrm{Cu}_{3} \mathrm{O}_{y} \quad$ film are shown in Fig. 5. The calculated critical exponent in vicinity of $T_{\mathrm{c}}$ temperature interval was determined to be $\lambda=0.36 \pm 0.05$. This means that in this temperature regime the true critical fluctuations play a dominant role according to 3 dimensional XY plane model.

The fluctuations of magnetoconductivity in $\left(\mathrm{Tl}_{0.5} \mathrm{~Pb}_{0.5}\right) \mathrm{Sr}_{2}\left(\mathrm{Ca}_{1-x} \mathrm{Gd}_{x}\right) \mathrm{Cu}_{2} \mathrm{O}_{z} \quad$ superconductor with $x=0.2$ have also been analysed. The relation 


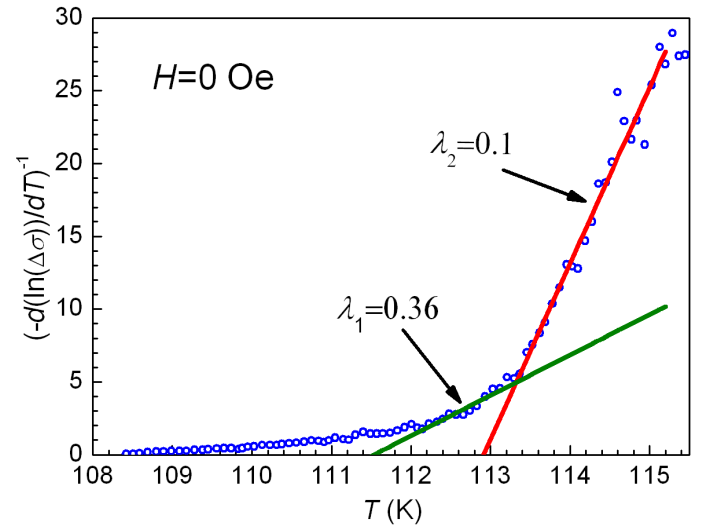

Fig. 5. Logarithmic $T$ derivative of $\Delta \sigma$ as a function of temperature of a $\left(\mathrm{Tl}_{0.6} \mathrm{~Pb}_{0.24} \mathrm{Bi}_{0.16}\right)\left(\mathrm{Ba}_{0.1} \mathrm{Sr}_{0.9}\right)_{2} \mathrm{Ca}_{2} \mathrm{Cu}_{3} \mathrm{O}_{y}$ film at the zero applied magnetic field. The solid lines correspond to fits to Eq. (19). The critical exponents $\lambda_{1}$ and $\lambda_{2}$ are calculated from the fit parameter of Eq. (19).

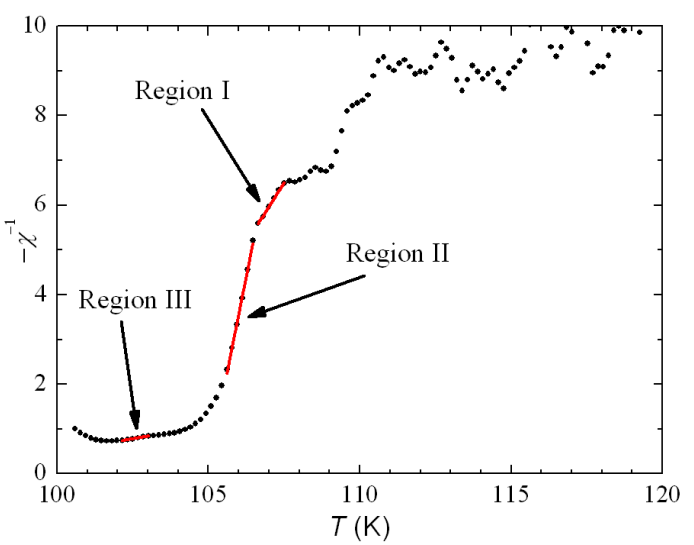

Fig. 6. Representative plot of $(-\mathrm{d}(\ln (\Delta \sigma)) / \mathrm{d} T)^{-1}$ vs. $T$ of $\left(\mathrm{Tl}_{0.5} \mathrm{~Pb}_{0.5}\right) \mathrm{Sr}_{2}\left(\mathrm{Ca}_{0.8} \mathrm{Gd}_{0.2}\right) \mathrm{Cu}_{2} \mathrm{O}_{y}$ without applied magnetic fields. There are three regions in which the linear fitting can be obtained (see text).

expressed by Eq. (19) for the sample with $x=0.2$ and for $H=0$ is shown in Fig. 6. The calculated temperature derivatives of the resistance for the sample with $x=0.2$ near the critical temperature are shown in Fig. 7. Two features have been noticed: the maximum associated with the critical fluctuation temperature $T_{\text {cf }}$ and the shoulder related to the temperatures below $T_{\text {cf }}$. The shoulder shifts to lower temperatures when the applied magnetic field increases, whereas the maximum does not depend on the magnetic field up to the maximal fields used in these experiments. Above $T_{\mathrm{cf}}$ the transition is dominated by superconducting fluctuation in the normal state in which two regions I and II were defined (region III is defined below the $T_{\text {cf }}$ ) (see Fig. 6).

The calculated field dependence of the critical exponents of $\left(\mathrm{Tl}_{0.5} \mathrm{~Pb}_{0.5}\right) \mathrm{Sr}_{2}\left(\mathrm{Ca}_{1-x} \mathrm{Gd}_{x}\right) \mathrm{Cu}_{2} \mathrm{O}_{z}$ superconductors with $x=0.2$ in the first and second regions are shown in Fig. 8. In the first region the critical exponent is equal $\lambda=0.93$ at $H=0$ Oe and decreases slowly with the rising of the magnetic field, finally reaching value of $\lambda=0.77$ at $H=1000$ Oe. It means that the critical exponents change by $12 \%$ within this magnetic field range. The standard deviation of the critical exponent in this region is equal $\Delta \lambda=0.16$. The dimensionalities of the fluctuating system, calculated using Eq. (18), were found to be $d=2.1$ and $d=2.5$, respectively. To summarize, one can conclude that in the region $\mathrm{I}$ the $2 \mathrm{D}$ fluctuating system exists and the applied magnetic field enhances this dimensionality. In the region II the critical exponent increases by $50 \%$ of its initial value when the magnetic field is risen up to 1000 Oe. In the region II the critical exponent is equal $\lambda=0.30$ at $H=0$ Oe and increases up to $\lambda=0.46$ at $H=1000$ Oe. The standard deviation of the critical exponent in this region is equal $\Delta \lambda=0.04$. Again, using Eq. (18) the dimensionalities of the fluctuating system have been found to be $d=3.4$ at $H=0$ Oe and $d=3.1$ at $H=1000$ Oe. It means that there is a $3 \mathrm{D}$ fluctuating system within the interval of the applied magnetic fields. For both regions only gaussian fluctuations are observed. Similar, in our previous paper [35] on the shape of specific heat anomaly around $T_{\mathrm{c}}$ in $\mathrm{DyBa}_{2} \mathrm{Cu}_{3} \mathrm{O}_{x}$ we have found a large effect of the gaussian fluctuations within a few Kelvins around $T_{\mathrm{c}}$. The calculated critical exponents in the region III, as a function of magnetic field are shown in Fig. 9. In this region the critical exponent is equal $\lambda=1.98$ at $H=0$ Oe and increases up to $\lambda=2.96$ at $H=1000$ Oe. In the region III the critical exponent increases by $50 \%$ of its initial value. The standard deviation of the critical exponent in this region is equal $\Delta \lambda=0.08$. This region is difficult to be clearly explained and understood. The authors of the paper [25] interpreted this behavior in terms of dissipative flux motion in the presence of the applied magnetic field. On the contrary some authors [36-38] suggest that there is a phase transition involving quenched disorder within the system of weak links. They claim that if the exponents are larger than of two (2.2-2.7) [38] the system shows the paracoherentcoherent transitions of the granular array, where the fluctuating phase of the order parameter in each grain becomes long-range ordered as a consequence of the activation of weak links between grains. The high values of the critical exponents near the zero resistance critical temperature suggest that the fluctuating Cooper pairs are forced to move along the one dimension percolation path. This idea was proposed in the paper [39] in which the crossover from $2 \mathrm{D}$ to $1 \mathrm{D}$ in the polycrystalline $\mathrm{Hg}-\mathrm{Tl}$ 1223 superconductors was observed. On the other hand the crossover from $2 \mathrm{D}$ to $3 \mathrm{D}$ dimensional fluctuation in the polycrystalline $\mathrm{Hg}-\mathrm{Tl} 1223$ superconductors was also noticed [40]. The measurements of the conductivity of $c$-axis oriented $\mathrm{Tl}-\mathrm{Ba}-\mathrm{Ca}-\mathrm{Cu}-\mathrm{O}(1223)$ and (2212) thin films in the paper [41] show only two-dimensional fluctuations without crossover to $3 \mathrm{D}$, whereas in $\mathrm{Tl}-\mathrm{Ba}-\mathrm{Ca}-$ $\mathrm{Cu}-\mathrm{O}$ (2212) single crystals [42] the crossover from 2D 
to 3D was observed near the transition temperature in some samples. The critical exponents evaluated for the temperatures below $T_{\mathrm{c}}$ (for instance, in the region III for the $\left(\mathrm{Tl}_{0.5} \mathrm{~Pb}_{0.5}\right) \mathrm{Sr}_{2}\left(\mathrm{Ca}_{0.8} \mathrm{Gd}_{0.2}\right) \mathrm{Cu}_{2} \mathrm{O}_{y}$ superconductor) characterize rather extrinsic behavior of the sample fabricated at certain preparation conditions but not the intrinsic physical properties of the superconducting material in general.

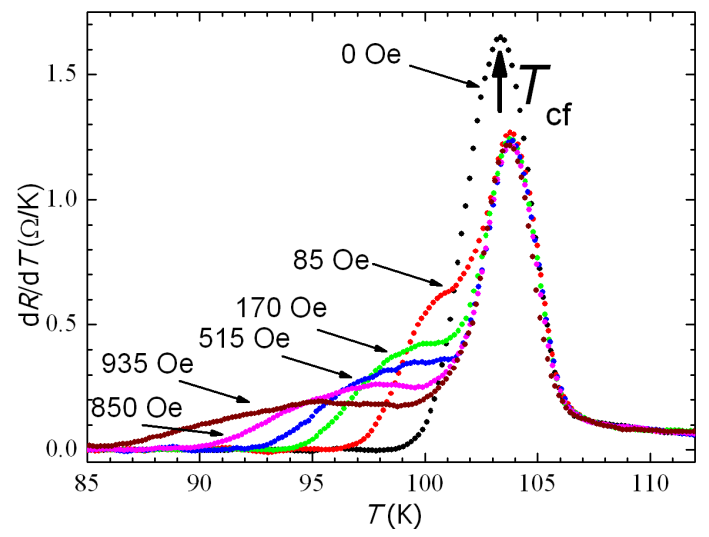

Fig. 7. Temperature derivatives of the resistance near the critical temperature for different values of the applied magnetic field of $\left(\mathrm{Tl}_{0.5} \mathrm{~Pb}_{0.5}\right) \mathrm{Sr}_{2}\left(\mathrm{Ca}_{0.8} \mathrm{Gd}_{0.2}\right) \mathrm{Cu}_{2} \mathrm{O}_{y}$ superconductor.

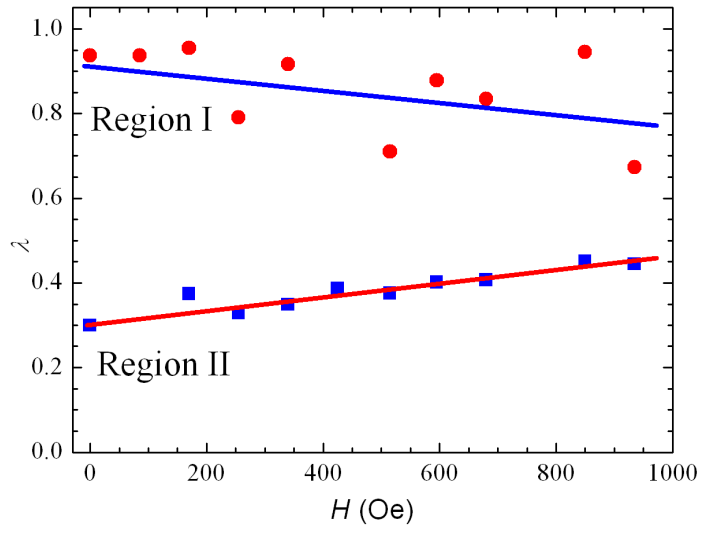

Fig. 8. Critical exponents as a function of the applied magnetic field of $\left(\mathrm{Tl}_{0.5} \mathrm{~Pb}_{0.5}\right) \mathrm{Sr}_{2}\left(\mathrm{Ca}_{0.8} \mathrm{Gd}_{0.2}\right) \mathrm{Cu}_{2} \mathrm{O}_{y} \mathrm{su}-$ perconductor for region I (closed circles) and for region II (closed squares) with linear fits.

\section{Summary}

The resistive transition from the normal to the superconducting state of the high temperature superconductors was characterized by its width, shape and by thermal fluctuations. The magnetically anomalous broadening of the resistive transition in the applied magnetic fields is well described by the Eq. (8). The shape of the

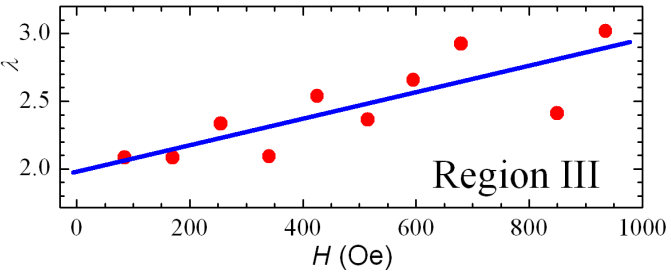

Fig. 9. Critical exponents as a function of the applied magnetic field of $\left(\mathrm{Tl}_{0.5} \mathrm{~Pb}_{0.5}\right) \mathrm{Sr}_{2}\left(\mathrm{Ca}_{0.8} \mathrm{Gd}_{0.2}\right) \mathrm{Cu}_{2} \mathrm{O}_{y} \mathrm{Su}_{-}$ perconductor for region III (closed circles) with linear fit.

resistive transition could be analyzed using two models: first one, based on the Ambegaokar-Halperin theory and the other one, based on Anderson-Kim theory. Because of very short coherence length and the large anisotropy of high temperature superconductors the thermal fluctuations around the superconducting transition were observed. The critical fluctuations were revealed at the temperatures below the critical temperature of fluctuations $T_{\mathrm{cf}}$, and the gaussian (stochastic) fluctuations were observed above $T_{\text {cf }}$. These fluctuations show the magnetic field dependence.

\section{Acknowledgments}

This work was supported by the Polish Ministry of Science and Higher Education and its grants for Scientific Research. One of us (M. Ch.) has been partly supported by the EU Human Capital Operation Program, Polish Project No. POKL.04.0101-00-434/08-00.

\section{References}

[1] M. Tinkham, Introduction to Superconductivity, 2nd edition, McGraw-Hill, Inc., 1996.

[2] T.T.M. Plastra, B. Batlogg, L.F. Schneemeyer, J.V. Waszczak, Phys. Rev. Lett. 61, 1662 (1988).

[3] T.T.M. Plastra, B. Batlogg, R.B. van Dover, L.F. Schneemeyer, J.V. Waszczak, Appl. Rev. Lett. 54, 763 (1988).

[4] K. Kadowaki, Y. Songlin, K. Kitazawa, Supercond. Sci. Technol. 7, 519 (1994).

[5] W.K. Kwok, U. Welp, G.W. Crabtree, K.G. Vandervoort, R. Hulscher, J.Z. Liu, Phys. Rev. Lett. 64, 966 (1990).

[6] V. Ambegaokar, B.I. Halperin, Phys. Rev. Lett. 22, 1364, (1969).

[7] M. Tinkham, Phys. Rev. Lett. 61, 1658 (1988).

[8] P.W. Anderson, Y.B. Kim, Rev. Mod. Phys. 36, 39 (1964).

[9] J.-J. Kim, H.-K. Lee, J. Chung, H.J. Shin, H.J. Lee, J.K. Ku, Phys. Rev. B 43, 2962 (1991).

[10] O. Heiml, G. Gritzner, Supercond. Sci. Technol. 15, 956 (2002).

[11] W.M. Woch, R. Zalecki, A. Kołodziejczyk, J. Chmist, O. Heiml, G. Gritzner, Acta Phys. Pol. A 106, 15, 785 (2004). 
[12] W.M. Woch, R. Zalecki, A. Kołodziejczyk, O. Heiml, G. Gritzner, Physica C 434, 17 (2006).

[13] C. Deinhofer, G. Gritzner, Supercond. Sci. Technol. 17, 1196 (2004).

[14] W.M. Woch, R. Zalecki, A. Kołodziejczyk, C. Deinhofer, G. Gritzner, J. Alloys Compd. 442, 209 (2007).

[15] W.M. Woch, R. Zalecki, A. Kołodziejczyk, C. Deinhofer, G. Gritzner, Acta Phys. Pol. A 111, 737 (2007).

[16] W.M. Woch, W. Tokarz, R. Zalecki, A. Kołodziejczyk, C. Deinhofer, G. Gritzner, Supercond. Sci. Technol. 23, 025004 (2010).

[17] H. Sudra, G. Gritzner, Physica C 443, 57 (2006).

[18] W.M. Woch, R. Zalecki, A. Kołodziejczyk, H. Sudra, G. Gritzner, Supercond. Sci. Technol. 21, 085002 (2008).

[19] W.M. Woch, R. Zalecki, A. Kołodziejczyk, H. Sudra, G. Gritzner, Mater. Sci. - Poland 26, 1091 (2008).

[20] W.M. Woch, J. Niewolski, W. Tokarz, A. Kołodziejczyk, H. Sudra, G. Gritzner, Acta Phys. Pol. A 118, 319 (2010).

[21] Y. Yeshurun, A.P. Malozemoff, Phys. Rev. Lett. 60, 2202 (1988)

[22] J.R.L. de Almeida, D.J. Thouless, J. Phys A 11, 983 (1978).

[23] K.A. Müller, M. Takashige, J. Bednorz, Phys. Rev. Lett. 58, 1143 (1987).

[24] W.M. Woch, M. Chrobak A. Kołodziejczyk, Acta Phys. Pol. A 118, 389 (2010).

[25] P. Pureur, R.M. Costa, P. Rodrigues, Jr., J. Schaf, J.V. Kunzler, Phys. Rev. B 47, 11420 (1993).

[26] R.M. Costa, P. Pureur, M. Gusmao, S. Senoussi, K. Behnia, Phys. Rev. B 64, 214513 (2001).

[27] R.M. Costa, L.M. Ferreira, P. Pureur, H.A. Borges, P. Lejay, Phys. Rev. B 69, 212505 (2004).

[28] D.H. Kim, K.E. Gray, M.D. Trochet, Phys. Rev. B 45, 10801 (1992).
[29] H.M. Duan, W. Kiehl, C. Dong, A.W. Cordes, M.J. Saeed, D.L. Viar, A.M. Hermann, Phys. Rev. B 43, 12925 (1991).

[30] N.H. Ahmad, N.A. Khan, A.K. Yahya, J. Alloys Compd. 492, 473 (2010), and references therein.

[31] L.G. Aslamazov, A.I. Larkin, Sov. Phys. Solid State 10, 875 (1968); A.I. Larkin, A.A. Varlamov, in: Superconductivity, Vol. 1 Eds. K.H. Bennemann, J.B. Ketterson, Springer-Verlag, Heidelberg 2008, p. 369; C.J. Lobb, Phys. Rev. B 36, 3930 (1987).

[32] V.I. Ginzgurg, L.D. Landau, Zh. Exp. Teor. Fiz. 20, 1064 (1950).

[33] A. Mohanta, D. Behera, Physica C 470, 295 (2010).

[34] J. Rosenblatt, P. Peyral, A. Raboutou, C. Lebeau, Physica B 152, 95 (1988).

[35] A. Kozłowski, Z. Tarnawski, A. Kołodziejczyk, J. Chmist, T. Ściężor, R. Zalecki, Physica C 184, 113 (1991).

[36] M.A. Grusniko, P.M. Mors, Phys. Rev. B 42, 10030 (1990).

[37] D.S Fischer, M.P.A. Fisher, D. House, Phys. Rev. B 43, 130 (1991).

[38] C. Lebeau, J. Rosenblatt, A. Raboutou, P. Peyral, Europhys. Lett. 1, 313 (1986).

[39] S.H. Han, J. Axnas, B.R. Zhao, O. Rapp, Physica $C$ 408-410, 679 (2004).

[40] S.H. Han, I. Bryntse, J. Axnas, B.R. Zhao, O. Rapp, Physica C 388-389, 349 (2003).

[41] D.H. Kim, A.M. Goldman, J.H. Kang, K.E. Gray, R.T. Kampwirth, Phys. Rev. B 39, 12275 (1989).

[42] H.M. Duan, W. Kiehl, C. Dong, A.W. Cordes, M.J. Saeed, D.L. Viar, A.M. Hermann, Phys. Rev. B 43, 12925 (1991). 